\title{
Generalized Hold Funtion Design For Periodically Time-Varying Systems
}

\author{
Min-Shin Chin \\ Department of Mechanical Engineering \\ National Taiwan University \\ Taipei, Taiwan, Republic of China \\ TEL:02-3630231 ext. 2414 \\ FAX:02-3631755 \\ EMAIL:mschen@ccms.ntu.edu.tw
}

\begin{abstract}
Most control designs for periodically time-varying systems use either full state feedback or observer-based state feedback. In this paper, it is shown that static output feedback is sufficient for the exponential stabilization of a periodical system under both the controllability and observability assumptions. In fact, by incorporating a new generalized hold function in the control design, one is able to arbitrarily shift all the Poincaré exponents of the periodical system. Most importantly, the control singal is guaranteed to be continuous in time while the control signal from previous designs may be discontinuous.
\end{abstract}

\section{Introduction}

An important class of linear time-varying systems in the physical world is the class of periodical systems, in which the system parameters vary periodically. Analysis for such systems has been done thoroughly in the past $[1,2]$. One of the most important results is summarized in the Floquet theory, which states that the stability property of a linear periodical system can be determined by $n$ constant numbers called the Poincaré exponents, where $n$ is the dimension of the system. If all the Poincare exponents are in the open left-half plane, the periodical system is exponentially stable. If at least one of the Poincare exponents is in the open right-half plane, the system is unstable.

For the stability synthesis of periodical systems, most control designs are based on the assumption that all the state variables are accessible for measurement. Among these, the earliest approach is the $L Q$ optimal control, in which one solves a periodical Riccati equation to obtain a stabilizing state feedback control $[3,4]$. Another approach is the modal control proposed in [5], which can arbitrarily shift only one of the Poincaré exponents of the system. Later, a layer of modal controllers are suggested to shift all the Poincaré exponents [6]. Recently, the generalized hold function design, originally developed in [7], is applied to the state feedback control of a periodical system [8]. However, the resultant control signal may have large discontinuities in time. In practice, such large discontinuities are either unacceptable under the actuator constraint or undesirable due to the possible excitation of high frequency unmodelled dynamics. Even though an attempt has been made to make the control signal continuous, its success is obstructed by a singularity problem [8].

In this paper, a new design is proposed to avoid discontinuities in the control signal. Furthermore, it is shown that when the periodical system is both controllable and observable, simple static output feedback control is sufficient for the arbitrary assignment of all the Poincaré exponents (note that full state feedback is required in [8]). The key elements in the new control design are the well-known Floquet transformation [5] and a new generalized hold function design [7]. This paper is arranged as follows. In Section 2, the definition of Poincaré exponents for a periodical system is presented. In Section 3, a discontinuous output feedback control is developed to assign the Poincaré exponents of the closed-loop system, and the control design is further modified in Section 4 in order to remove discontinuities in the control signals.

\section{Stability Analysis for Periodical Systems}

Consider the stability analysis of the following system

$$
\dot{x}(t)=A(t) x(t)
$$

where $x(t) \in R^{n}$ is the state vector, the system matrix 
$A(t) \in R^{n \times n}$ is T-periodic in the sense that

$$
A(t+T)=A(t), \quad \forall t>0 .
$$

In the famous Floquet theory [1], the stability property of (1) is studied through a state transformation into a new coordinate, on which the system matrix becomes time invariant. Such a transformation, called the Floquet transformation, is given by

$$
z(t)=P(t) x(t), \quad P(t)=e^{J t} \Phi^{-1}(t, 0),
$$

where $\Phi(t, 0)$ is the state transition matrix [2] of (1), and $J$ is a constant matrix given by

$$
J=\frac{1}{T} \ln \Phi(T, 0) .
$$

From (1), (2) and (3), the periodical system (1) has a constant representation in the new coordinate:

$$
\dot{z}(t)=J z(t) .
$$

One can verify (see [2]) that the state transformation matrix $P(t)$ in (2) is also T-periodic, and remains uniformly bounded and nonsingular. The stability property of the periodical system (1) can then be inferred from that of the constant system (5). In the literature, the eigenvalues of the constant matrix $J$ in (5) are referred as the Poincaré (or characteristic) exponents

$$
\text { P.E. } \triangleq \lambda_{i}(J)=\frac{1}{T} \ln \lambda_{i}[\Phi(T, 0)]
$$

where the second equality results from (3). The condition for exponential stability of (1) is thus

$$
\operatorname{Re}\left[\lambda_{i}(J)\right]<0
$$

or equivalently,

$$
\left|\lambda_{i}[\Phi(T, 0)]\right|<1,
$$

due to (5), where $\Phi(T, 0)$ is called the monodromy matrix [8]. To end this Section, note that from (2) and (3) one can derive the identity

$$
\Phi(T, t)=e^{J(T-t)} P(t),
$$

which will be used in subsequent Sections.

\section{$3 \quad$ Stability Synthesis by Discontinuous Control}

Consider now a periodical system with control

$$
\begin{aligned}
& \dot{x}(t)=A(t) x(t)+B(t) u(t), \quad x(0)=x_{0}, \\
& y(t)=C(t) x(t),
\end{aligned}
$$

where $x(t) \in R^{n}$ is the state vector, $u(t) \in R^{p}$ the control input, and $y(t) \in R^{q}$ the system output. It is assumed that the only accessible signal is the systern output $y(t)$, and that $A(t) \in R^{n \times n}, B(t) \in R^{n \times p}, C(t) \in$ $R^{q \times n}$ are all $\mathrm{T}$-periodic; i.e.,

$(A(t+T), B(t+T), C(t+T))=(A(t), B(t), C(t)) \quad \forall t>0$.

The objective in this Section is to find a stabilizing static output feedback control $u(t)$ that can arbitrarily assign the locations of the Poincaré exponents of the closedloop system. For this purpose, the following assumptions are required:

(A1) The pair $(A(t), B(t))$ is controllable in the sense that its controllability grammian [1] $W$ defined on the time interval $[0, T]$ is positive definite, where

$$
W=\int_{0}^{T} \Phi(T, \tau) B(\tau) B^{T}(\tau) \Phi^{T}(T, \tau) d \tau
$$

and $\Phi(t, \tau)$ is the open-loop state transition matrix of (1).

(A2) The constant pair $\left(\Phi(T, 0), C_{0}\right)$ is observable [9], where $\Phi(T, 0)$ is the open-loop monodromy matrix, and $C_{0} \triangleq C(0)=C(k T)$.

The proposed control design proceeds as follows.

Step I. Choose $n$ Poincaré exponents $\omega_{i}$ (real or in complex conjugate pairs) with $\operatorname{Re}\left(\omega_{i}\right)<0$. Calculate the eigenvalues for the closed-loop monodromy matrix based on (5):

$$
\lambda_{i}^{c}=e^{T \omega_{i}}
$$

and then use the pole placement algorithm [9] to find a constant matrix $L \in R^{n \times q}$ so that

$$
\lambda_{i}\left(\Phi(T, 0)+L C_{0}\right)=\lambda_{i}^{c}
$$

where $\Phi(T, 0)$ and $C_{0}$ are as in Assumption (A2). Note that Assumption (A2) guarantees the existence of $L$ in (12) for any choice of the value $\lambda_{i}^{c}$.

Step II. Construct a T-periodic generalized hold function $G(t) \in R^{p \times q}$ with $G(t+T)=G(t)$, and

$$
G(t)=B^{T}(t) \Phi^{T}(T, t) W^{-1} L, \quad t \in[0, T),
$$

where $\Phi(T, t)$ and $W$ are as in (9). Note that Assumption (A1) guarantees the invertibility of $W$.

Step III. Set the control input to be

$$
u(t)=G(t) y(k T), \quad t \in[k T, k T+T),
$$

where $y(k T)$ is the sampled system output with a sampling period $T$, and $G(t)$ is in (12). 
Notice that in the above control design, one needs to calculate the open-loop state transition matrix $\Phi(T, \tau)$ over the entire period $\tau \in[0, T]$. Such computation becomes difficult when the period is long or when the system dimension is large. For an efficient and accurate numerical algorithm in calculating the state transition matrix, one may refer to $[10,11]$.

Theorem 1 : Under the Assumptions (A1) and (A2), the static output feedback control (13) stabilizes the system (8) exponentially. Furthermore, the closed-loop Poincare exponents are located as specified in Step $\mathbf{I}$ in the design procedure.

A simple simulation example is provided below to verify the proposed control design.

Example 1 : Consider a periodic system, which is open-loop unstable,

$$
\begin{gathered}
\dot{x}(t)=\left[\begin{array}{cc}
1+\cos 2 \pi t & 0 \\
0 & 2+\sin 2 \pi t
\end{array}\right] x(t)+\left[\begin{array}{r}
1 \\
-1
\end{array}\right] u(t), \\
y(t)=\left[\begin{array}{ll}
2+\sin 4 \pi t & -9+\sin 4 \pi t
\end{array}\right] x(t) .
\end{gathered}
$$

The period of the system is one second. The proposed control (13) is applied to the system with the initial condition $x^{T}(0)=[2,2]$. The design parameters in Step I are $\omega_{1}=\ln 0.1$ and $\omega_{2}=\ln 0.3$. Figure 1 shows the time history of the system state, which converges exponentially as predicted by Theorem 1, and Figure 2 shows the control input.

\section{Stability Synthesis by Continuous Control}

Figure 2 in Example 1 reveals a problem with the control design based on the generalized hold function: the control signal $u(t)$ in (13) has large discontinuities at the sampling instants $t=k T$. Since control with large discontinuities is either not implementable in practice or unacceptable from the robustness consideration, the objective of this Section is to modify the previous control design so that assignment of the Poincaré exponents can be achieved by a continuous control input.

The approach adopted here is to find a new generalized hold function $\bar{G}(t)$ to replace $G(t)$ in the discontinuous control $(13)$, with $\bar{G}(t)$ now satisfying

$$
\bar{G}(k T)=0, \quad \forall k=0,1,2, \ldots
$$

This will force the control input $u(t)$ to be continuous at any time instant $t=k T$; in fact, according to (13) and (14), one has

$$
u\left(t^{-}\right)=u\left(t^{+}\right)=0, \quad \text { at } t=k T .
$$

The modified design procedure is as follows.

Step I and Step II are the same as in the previous Section.

Step III. Choose any two T-periodic matrix functions $G_{1}(t) \in R^{p \times q}$ and $G_{2}(t) \in R^{p \times q}$ that are continuous on $(0, T)$. Calculate $\mathbf{R}\left[G_{1}(\tau)\right]$ and $\mathbf{R}\left[G_{2}(\tau)\right]$, where $\mathbf{R}[\cdot]$ is the controllability map

$$
\mathbf{R}\left[G_{i}(\tau)\right]=\int_{0}^{T} \Phi(T, \tau) B(\tau) G_{i}(\tau) d \tau
$$

Denote the two resultant constant matrices by

$L_{1} \triangleq \mathbf{R}\left[G_{1}(\tau)\right] \in R^{n \times q}, \quad L_{2} \triangleq \mathbf{R}\left[G_{2}(\tau)\right] \in R^{n \times q}$

Step IV. Construct the following two T-periodic matrix functions from $G_{1}(t)$ and $G_{2}(t), t \in[0, T)$,

$$
\begin{aligned}
& G_{01}(t)=G_{1}(t)-B^{T}(t) \Phi^{T}(T, t) W^{-1} L_{1} \\
& G_{02}(t)=G_{2}(t)-B^{T}(t) \Phi^{T}(T, t) W^{-1} L_{2}
\end{aligned}
$$

where $B(t), \Phi(T, t)$, and $W$ are as in (12), and $L_{1}$ and $L_{2}$ in (16).

Step V. Case (a). When the number of inputs are no less than that of outputs $(p \geq q)$, determine two constant matrices $\alpha_{1}$ and $\alpha_{2} \in R^{p \times p}$ from the equations

$$
\begin{array}{r}
G\left(0^{+}\right)+\alpha_{1} G_{01}\left(0^{+}\right)+\alpha_{2} G_{02}\left(0^{+}\right)=0, \\
G\left(T^{-}\right)+\alpha_{1} G_{01}\left(T^{-}\right)+\alpha_{2} G_{02}\left(T^{-}\right)=0
\end{array}
$$

where $G(t)$ is given by (12), and $G_{01}(t)$ and $G_{02}(t)$ by (17) and (18). Solutions $\alpha_{1}$ and $\alpha_{2}$ in (19) and (20) exist if the following matrix is full rank

$$
\operatorname{rank}\left|\begin{array}{ll}
G_{01}\left(0^{+}\right) & G_{01}\left(T^{-}\right) \\
G_{02}\left(0^{+}\right) & G_{02}\left(T^{-}\right)
\end{array}\right|=2 q
$$

If this condition is not satisfied for the $G_{1}(t)$ and $G_{2}(t)$ chosen in Step III, one can simply choose a different pair of $G_{1}(t)$ and $G_{2}(t)$ until the required rank condition is satisfied. Notice that there are infinite degrees of freedom in choosing $G_{1}(t)$ and $G_{2}(t)$ for no constraint is imposed on them except continuity on the interval $(0, T)$. Hence, choosing $G_{1}(t)$ and $G_{2}(t)$ to meet the above rank condition is in general quite easy.

Case (b). When the number of inputs are no more than that of outputs $(p \leq q)$, determine two constant matrices $\beta_{1}$ and $\beta_{2} \in R^{q \times q}$ from the equations

$$
\begin{aligned}
& G\left(0^{+}\right)+G_{01}\left(0^{+}\right) \beta_{1}+G_{02}\left(0^{+}\right) \beta_{2}=0, \\
& G\left(T^{-}\right)+G_{01}\left(T^{-}\right) \beta_{1}+G_{02}\left(T^{-}\right) \beta_{2}=0,
\end{aligned}
$$

where $G(t), G_{01}(t)$ and $G_{02}(t)$ are as in (19). Similarly, it will be assumed that in solving the above equations for 
$\beta_{1}$ and $\beta_{2}$, the following matrix (note that it is different from the previous one) is full rank

$$
\operatorname{rank}\left|\begin{array}{cc}
G_{01}\left(0^{+}\right) & G_{02}\left(0^{+}\right) \\
G_{01}\left(T^{-}\right) & G_{02}\left(T^{-}\right)
\end{array}\right|=2 p .
$$

Step VI. Set the control input to be

$$
u(t)=\bar{G}(t) y(k T), \quad t \in[k T, k T+T),
$$

where $y(k T)$ is the sampled system output, and $\vec{G}(t)=$ $\bar{G}(t+T)$ is the new generalized hold function

$$
\begin{array}{lll}
\bar{G}(t)=G(t)+\alpha_{1} G_{01}(t)+\alpha_{2} G_{02}(t), & \text { if } & p \geq q \\
\bar{G}(t)=G(t)+G_{01}(t) \beta_{1}+G_{02}(t) \beta_{2}, & \text { if } \quad p \leq q,
\end{array}
$$

in which $G(t), G_{01}(t), G_{02}(t)$ are as in (19), and $\alpha_{i}$ and $\beta_{i}$ from Step V.

The following theorem shows that the new control (21) will shift the Poincaré exponents to the same desired locations as the discontinuous control (13) in the previous Section; furthermore, the new control input (21) is now continuous for all $t>0$.

Theorem 2 : The closed-loop system (8) with the continuous control (21) is exponentially stable. Furthermore, the closed-loop Poincaré exponents are located as specified in Theorem 1.

Example 2 : In this Example, the continuous control (21) is simulated for the same system as in Example 1. The control design parameters in Step I are as before, and the $\mathrm{T}$-periodic functions ( $\mathrm{T}=1$ second) in Step III are chosen to be $G_{1}(t)=t, G_{2}(t)=1-t$, where $t \in$ $[0, T)$. Figure 3 shows the time history of the controlled system state, and Figure 4 the control input. Observe that the control input now becomes continuous in time while the state convergence rate remains the same as in Example 1.

\section{References}

[1] E. A. Coddington and N. Levinson, Theory of Ordinary Differential Equations, McGraw Hill, New York, 1955.

[2] F. Callier and C. A. Desoer, Linear System Theory, Springer-Verlag, Hong Kong, 1992.

[3] S. Bittanti, P. Colaneri, and G. Guardabassi, "Analysis of the periodic Liapunov and Riccati equations via canonical decomposition," SIAM. J. Control and Optimization, vol. 24, pp.1138-1149, 1986.

[4] H. Kano, and T. Nishimura, "Periodic solutions of matrix Riccati equations with dectectability and stabilzability," Int. J. Control, vol. 29, pp.471-487, 1979.
[5] R. A. Calico, and W. E. Wiesel, "Control of timeperiodic systems," J. Guidance, vol. 7, pp.671-676, 1984.

[6] S. G. Webb, R. A. Calico, and W. E. Wiesel, "Timeperiodic control of a multiblade helicopter," J. Guidance, vol. 14, pp.1301-1308, 1991.

[7] A. B. Chammas, and C. T. Leondes, "On the design of linear time invariant systems by periodic output feedback, Part I and II," Int. J. Control, vol. 27, pp.885-903, 1978.

[8] P. T. Kabamba, "Monodromy eigenvalue assignment in linear periodic systems," IEEE Trans. on Automatic Control, AC-31, pp.950-952, 1986.

[9] T. Kailath, Linear Systems, Prentice-Hall, Englewood Cliffs, New Jersey, 1980.

[10] S. C. Sinha, and D-H, Wu, "An efficient computational scheme for the analysis of periodic systems," $J$. Sound and Vibration, vol. 151, pp.91-117, 1991.

[11] S. C. Sinha, D-H, Wu, V, Juneja, and P. Joseph, "Analysis of dynamic systems with periodically varying parameters via Chebyshev polynomials," J. Vibration and Acoustics, vol. 115, pp.96-102, 1993. 


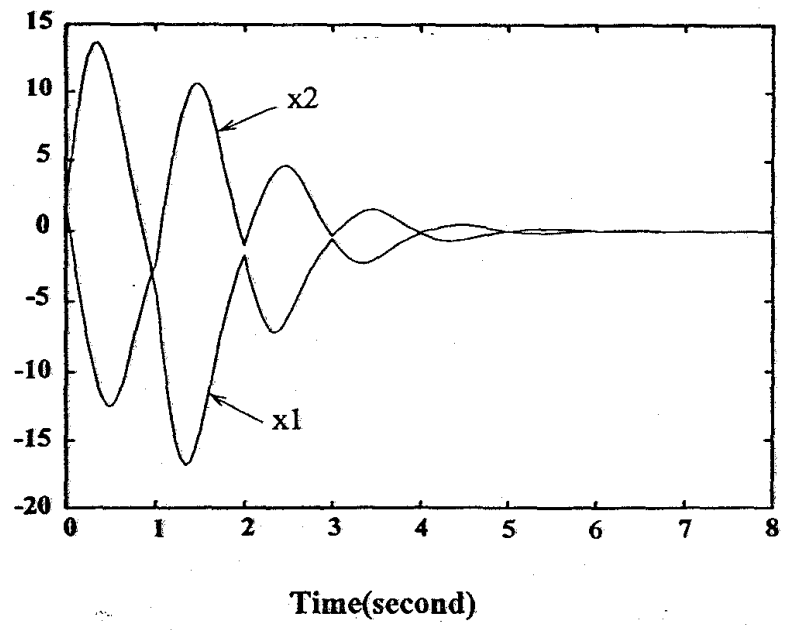

Figure 1. State response with discontinuous control

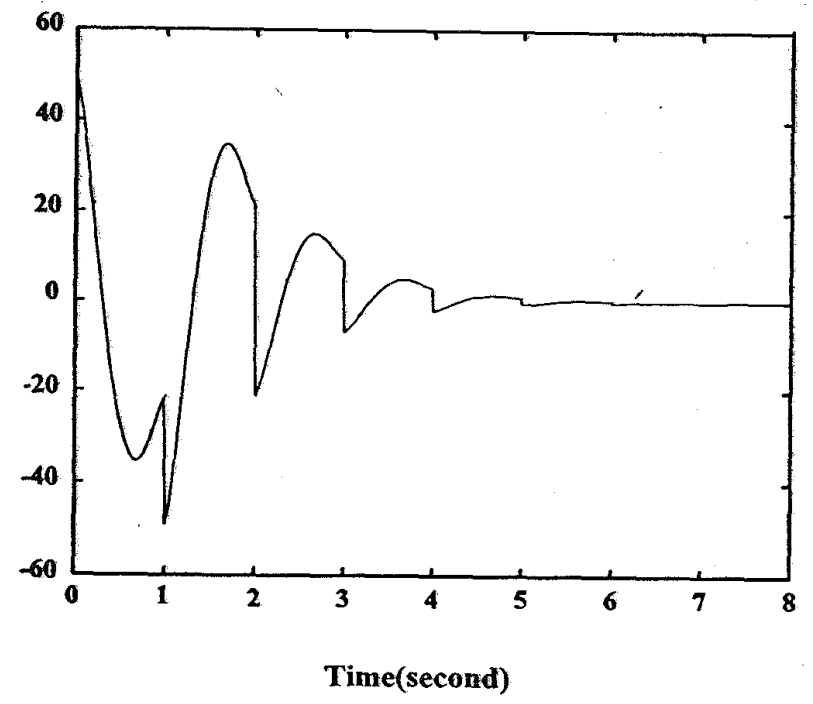

Figure 2. Control signal with discontinuous control

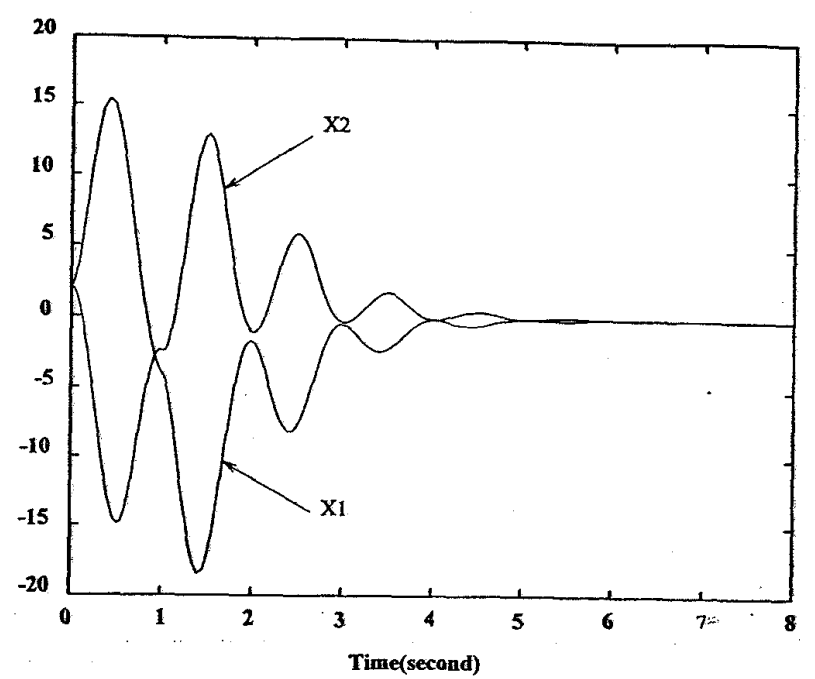

Figure 3. State response with continuous control

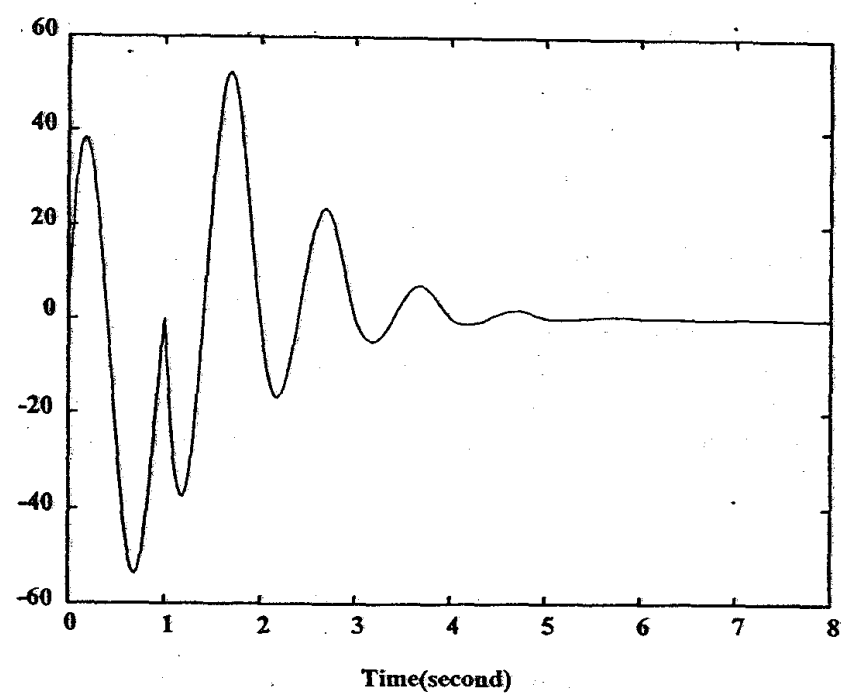

Figure 4. Control signal with continuous control 